\title{
PREENVELOPING CLASSES OF ACTS
}

\author{
MOHANAD FARHAN HAMID \\ Department of Production and Metallurgy Engineering, \\ University of Technology-Iraq, Baghdad, Iraq \\ E-mails:70261@uotechnology.edu.iq \\ mohanadfhamid@yahoo.com
}

\begin{abstract}
Preenvelopes of acts over a monoid are defined by analogy with Enochs' definition of preenvelopes of modules. Provided that it is closed for pure subacts, a class of acts is shown to be preenveloping precisely when it is closed under direct products. Examples of such classes include absolutely pure, weakly f-injective and weakly p-injective acts.
\end{abstract}

Key words and phrases. Pure subact, preenvelope, absolutely pure act, weakly f-injective act, weakly p-injective act.

\section{Introduction}

Acts and $S$-acts are always right unitary over a monoid $S$. Consider systems of equations over an act $A$ (with constants from $A$ ). Such equations are of one of the following forms

$$
x r=x s, x r=y s, \text { or } x r=a
$$

with $r$ and $s \in S, a \in A$ and indefinite $x$ and $y$. Let $A$ be a subact of an $S$-act $B$. If every finite such system of equations over $A$ that is solvable in $B$ has a solution in $A$, we say that $A$ is a pure subact of $B$. An act is called absolutely pure if it is pure in every containing act. This is equivalent to saying that $A$ is injective with respect to all inclusions $K \rightarrow L$ with $L$ a finitely presented and $K$ a finitely generated $S$-act 9 . If we assume that $L$ is cyclic in the above statement, we get the concept of almost pure acts [7. For a cardinal $\alpha$, an act is called $\alpha$-injective [6] if it is injective with respect to all inclusions $I \rightarrow S$ where $I$ is a right ideal of $S$ the cardinality of which is less than $\alpha$. If $\alpha=\aleph_{0}(\alpha=2)$ we get the concept of weakly f-injective (weakly p-injective) acts, respectively [6]. For any background information or undefined terms about acts over a monoid the reader is referred to [1, 8.

Preenvelopes and precovers of modules were introduced by E. Enochs 4 as generalizations of injective envelopes and projective covers, respectively. Analogously, they can be defined for act categories as follows. Let $\mathscr{C}$ be a class of acts over a monoid $S$ and $A$ an $S$-act. A map $\varphi: A \rightarrow C$, with $C \in \mathscr{C}$, is called a $\mathscr{C}$-preenvelope of $A$ if for any map $f: A \rightarrow C^{\prime}$, with $C^{\prime} \in \mathscr{C}$, there is a map $g: C \rightarrow C^{\prime}$ such that

1991 Mathematics Subject Classification. 20M30, 20M50. 
$g \circ \varphi=f$. A $\mathscr{C}$-envelope of $A$ is a $\mathscr{C}$-preenvelope with the added condition that if $C=C^{\prime}$ and $f=\varphi$ then $g$ must be an automorphism. Hence, a $\mathscr{C}$-envelope of an act, if it exists, is unique up to isomorphism. The class $\mathscr{C}$ is called (pre)enveloping if every act admits a $\mathscr{C}$-(pre)envelope. Covers and precovers are defined dually (see, e.g. 2]). Injective envelopes, defined in [3], are easily seen to be $\mathscr{I}$-envelopes, where $\mathscr{I}$ is the class of injective $S$-acts. It is easy to see that if the class $\mathscr{C}$ contains the injectives then $\mathscr{C}$-preenvelopes are monic.

The set-theoretic approach to the existence of preenvelopes of acts is adapted from [5] and [10].

\section{Preenvelopes of Acts}

A sufficient condition for the existence of preenvelopes is given in the following proposition. In what follows, $|X|$ means the cardinality of a set $X$.

Proposition 2.1. Let $A$ be an $S$-act and $\mathscr{C}$ a class of $S$-acts closed under direct products. Suppose that there is an infinite cardinal $\aleph_{\alpha}$ such that for every $C \in \mathscr{C}$ and every subact $T$ of $C$ with cardinality not exceeding $|A|$ there exists a subact $B$ of $C$ containing $T$ such that $B \in \mathscr{C}$ and $|B| \leq \aleph_{\alpha}$. Then $A$ has a $\mathscr{C}$-preenvelope.

Proof. Fix a set $X$ with cardinality $\aleph_{\alpha}$. If we can prove that any map $f: A \rightarrow C$, where $C \in \mathscr{C}$, can be factored through some $B \in \mathscr{C}$ such that $|B| \leq \aleph_{\alpha}$, then we can make a copy of $B$ with elements from the above set $X$. Hence, the totality of all maps $A \rightarrow B$ in the factorization $A \rightarrow B \rightarrow C$ is in fact a set, and the map $A \rightarrow \prod B$ is a $\mathscr{C}$-preenvelope. But for the map $f: A \rightarrow C$, we have $T=f(A)$ and $|T| \leq|A|$. Such $T$ is included, by assumption, in a $B$ as above, and we get our desired factorization $A \rightarrow B \rightarrow C$.

Before proceeding to our main result, we need the following lemma, which shows that between any act and a subact, we can always find a pure one whose cardinality depends only on that of the monoid $S$ and the subact.

Lemma 2.2. Let $T$ be a subact of an $S$-act $A$. Then there exist a cardinal $\aleph_{\alpha}$ depending on the cardinalities of $S$ and $T$, and a pure subact $U$ of $A$ containing $T$ such that $|U| \leq \aleph_{\alpha}$.

Proof. Let $\aleph_{\alpha}=\max \left\{\aleph_{0},|S|,|T|\right\}$. Consider the set $\Sigma$ of all finite systems of equations of the form

$$
x_{i} r_{i}=x_{i} s_{i}, y_{j} r_{j}^{\prime}=z_{j} s_{j}^{\prime}, w_{k} r_{k}^{\prime \prime}=t_{k} ; 1 \leq i \leq l, 1 \leq j \leq m, 1 \leq k \leq n
$$

with $r$ 's and $s$ 's $\in S, t$ 's $\in T$ and indefinite $x$ 's, $y$ 's, $z$ 's, and $w$ 's, that are solvable in $A$. For each variation of $l, m$, and $n$ we have a subset $\Sigma_{l, m, n}$ of $\Sigma$ with cardinality $|S|^{l} \cdot|S|^{l} \cdot|S|^{m} \cdot|S|^{m} \cdot|S|^{n} \cdot|T|^{n}=|S|^{2 l+2 m+n} \cdot|T|^{n} \leq \aleph_{\alpha}^{2 l+2 m+n} . \aleph_{\alpha}^{n}=\aleph_{\alpha}$. Therefore, the cardinality of $\Sigma$ is $|\Sigma| \leq \aleph_{0} . \aleph_{\alpha}=\aleph_{\alpha}$.

Put $U_{0}=T$ and consider the set $X_{1}$ of all systems in $\Sigma$ above with constants from $U_{0}$ that are solvable in $A$. For each such system, choose one solution. Let $\overline{X_{1}}$ be the set of these solutions. Therefore, $\left|\overline{X_{1}}\right| \leq|\Sigma| \leq \aleph_{\alpha}$. Now let $U_{1}=U_{0} \cup \overline{X_{1}} S$. Hence $\left|U_{1}\right| \leq\left|U_{0}\right|+\left|\overline{X_{1}}\right||S| \leq \aleph_{\alpha}+\aleph_{\alpha} . \aleph_{\alpha}=\aleph_{\alpha}$. Similarly, we construct the acts $U_{2}, U_{3}, \cdots$, each of which having cardinality not exceeding $\aleph_{\alpha}$ such that $U_{0} \subseteq U_{1} \subseteq U_{2} \subseteq \cdots \subseteq A$ and that every finite system of equations over $A$ that is solvable in $U_{n}$ has a solution in $U_{n+1}$. So, putting $U=\bigcup_{n=0}^{\infty} U_{n}$, we see that any finite system of equations with constants from $U$ that is solvable in $A$ must have its 
constants in some $U_{n}$. Therefore the system must be solvable in $U_{n+1} \subseteq U$. This proves that $U$ is pure in $A$.

Now for $|U|$. We know that $U=\bigcup_{n=0}^{\infty} U_{n}$, and therefore $|U| \leq\left|U_{0}\right|+\left|U_{1}\right|+\cdots \leq$ $\aleph_{\alpha}+\aleph_{\alpha}+\cdots=\aleph_{0} . \aleph_{\alpha}=\aleph_{\alpha}$.

Theorem 2.3. Let $\mathscr{C}$ be a class of acts closed for pure subacts. Then, $\mathscr{C}$ is preenveloping if and only if it is closed for direct products.

Proof. Sufficiency: let $A$ and $C$ be $S$-acts such that $C \in \mathscr{C}$ and let $T$ be a subact of $C$ with $|T| \leq|A|$. By lemma 2.2 and its proof, there is a pure subact $U$ of $C$ containing $T$ with $|U| \leq \max \left\{\aleph_{0},|T|,|S|\right\} \leq \max \left\{\aleph_{0},|A|,|S|\right\}$ and, by assumption, $U \in \mathscr{C}$. So, putting $\aleph_{\alpha}=\max \left\{\aleph_{0},|A|,|S|\right\}$, we see that $|U| \leq \aleph_{\alpha}$ and that $U$ is the act $B$ of proposition 2.1. Hence $A$ has a $\mathscr{C}$-preenvelope by proposition 2.1 Necessity: let $\left\{C_{i}\right\}$ be a family of acts in $\mathscr{C}$ and let $f: \prod C_{i} \rightarrow C$ be a $\mathscr{C}$-preenvelope of $\prod C_{i}$. This means that for every $C_{i}$ there is a $\lambda_{i}: C \rightarrow C_{i}$ such that $\lambda_{i} f=\pi_{i}$, where $\pi_{i}$ is the projection of $\prod C_{i}$ onto $C_{i}$ for each $i$. Hence, there is a (unique) $\lambda: C \rightarrow \prod C_{i}$ such that $\pi_{i} \lambda=\lambda_{i}$ for all $i$. But then $\lambda f$ is the identity map of $\prod C_{i}$, and $\prod C_{i}$ is a retract, hence a pure subact, of $C$. Therefore, $\prod C_{i} \in \mathscr{C}$.

Corollary 2.4. Each of the classes of absolutely pure, almost pure, weakly finjective and weakly p-injective acts is preenveloping. Such preenvelopes are all monomorphisms.

Proof. It is easily seen that the above classes are closed for direct products and pure subacts. Therefore, by Theorem 2.3, they are all preenveloping classes. Moreover, as each class of them contains the injectives as a subclass, such preenvelopes must then be monic.

\section{REFERENCES}

1. Ahsan, J., Zhongkui, L.: A Homological Approach to the Theory of Monoids. Science Press, Beijing (2008).

2. Bailey, A., Renshaw, J.: A short note on strongly flat covers of acts over monoids, Semigroup Forum, 93(2), 416-422 (2016).

3. Berthiaume, P.: The injective envelopes of S-sets, Canad. Math. Bull. 10 (2), 261-273 (1967).

4. Enochs, E.: Injective and flat covers, envelopes and resolvents, Israel J. Math. 39, 189-209 (1981).

5. Enochs, E., Jenda, O.: Relative Homological Algebra. De Gruyter Expositions in Mathematics 30, Berlin 2000.

6. Gould, V.: The characterization of monoids by properties of their S-systems, Semigroup Forum, 32, 251-265 (1985).

7. Gould, V.: Completely right pure monoids, Proc. R. Ir. Acad., 87A, 73-82 (1987).

8. Kilp, M., Knauer, U., Mikhalev, A.: Monoids, Acts and Categories. Walter de Gruyter, Berlin (2000).

9. Normak, P.: Purity in the category of $M$-sets, Semigroup Forum, 20, 157-170 (1980).

10. Rada, J., Saorín, M.: Rings characterized by (pre)envelopes and (pre)covers of their modules, Comm. in Algebra, 26 (3), 899-912 (1998). 